\section{Severe developmental delay and epilepsy in a Japanese patient with severe congenital neutropenia due to HAX1 deficiency}

HAX1 deficiency has recently been identified as a cause of severe congenital neutropenia (SCN), but little is known about the phenotype. We described an SCN patient with a homozygous 256C-to- $T$ transition causing an $R 86 \mathrm{X}$ mutation in the HAX1 gene. Notably, the patient has been complicated by epilepsy and severe delay of motor, cognitive, and intellectual development; each developmental quotient was 21-26 at 7 years old. Growth failure and dental development delay were also noted. Neurodevelopmental delay in this patient expands the clinical phenotype of HAX1 deficiency and suggests an important role of HAX1 on neural development as well as myelopoiesis.

Haematologica 2007; 92:e123-e125 D0l: 10.3324/haematol.11973

Severe congenital neutropenia $(\mathrm{SCN})$ is a rare disorder of myelopoiesis resulting in recurrent life-threatening infections due to a lack of neutrophils. ${ }^{1}$ Individuals with SCN have characteristic bone marrow findings of myeloid hypoplasia with arrest of myelopoiesis at the promyelocyte/myelocyte stage. ${ }^{1}$ This disorder was first described in Kostmann's seminal paper in $1956 .{ }^{2}$ Recent evidence has indicated that SCN is a heterogeneous disorder involving mutations in various genes, including those encoding granulocyte colony stimulating factor receptor (G-CSFR) (CSF3R), ${ }^{3}$ neutrophil elastase (ELA2), ${ }^{4}$ Wiskott-Aldrich syndrome protein (WAS), ${ }^{5}$ and GFI-1. ${ }^{6}$ More recently, Klein et al. identified homozygous HAX1 mutations as a cause of an autosomal recessive SCN in 20 Middle Eastern patients and 3 classical Kostmann family members. ${ }^{7}$ They suggested that HAX1 is critical for maintaining the inner mitochondrial membrane potential and protecting against apoptosis in myeloid cells. ${ }^{7}$

HAX1 has been initially described as an interacting partner with HS-1, a signal-transduction protein in hematopoietic cells. ${ }^{8}$ HAX1 mRNA is ubiquitously expressed, ${ }^{8,9}$ and therefore its deficiency could theoretically result in dysfunction of several organs other than hematopoietic systems. Given that the precise function of HAX1 is not fully defined, clinical phenotypes of patients with deficiency of the responsible gene may be helpful. Here, we described an SCN patient caused by homozygous R86X mutations in HAX1, who presented with severe developmental delay followed by refractory epilepsy. This case may expand the phenotype of HAX1 deficiency in human and may provide a novel function of this gene.

\section{Design and Methods}

A 9-year-old boy was investigated because of congenital neutropenia with presentation of fulminant left otomastoiditis at two months old. The patient was born without asphyxia from nonconsanguineous parents at
39 weeks' gestation. There was no family history of immunodeficiency. Initial investigations demonstrated an absolute neutrophil count (ANC) of $0-0.09 \times 10^{9} / \mathrm{L}$ and marked monocytosis. Bone marrow examinations showed myeloid hypoplasia with maturation arrest at the promyelocyte/myelocyte stage. Cytogenetic analysis was normal. Under the diagnosis of SCN and otomastoiditis, the patient was treated with parenteral antibiotics and G-CSF. A higher dose of G-CSF at 10 $\mu \mathrm{g} / \mathrm{kg} /$ day for 9 days was required to achieve ANC over $1 \times 10^{9} / \mathrm{L}$. He was discharged with hearing impairment at a threshold of $50 \mathrm{~dB}$ on audiograms of the left ear. Details on his early clinical course were published previously. ${ }^{10}$

After discharge, he was treated with subcutaneous administration of G-CSF at $5-10 \mu \mathrm{g} / \mathrm{kg}$ two to three times per week and prophylactic use of sulfamethoxazole/trimethoprim. Six months after discharge, the frequency of G-CSF administration was reduced to once per week. Although ANC has remained low at 0.05$0.1 \times 10^{\circ} / \mathrm{L}$ under this therapy, there has not been any recurrent, invasive bacterial infection for more than 9 years. Annual follow-up bone marrow examinations have not shown any findings compatible with myelodysplastic syndrome or acute myelogenous leukemia.

Neurological development of the patient has been delayed. Months at which the patient achieved each developmental milestone were as follows; head control (4 months), sit alone (9 months), stand with support (12 months), stand without support (15 months), walk alone (18 months), and speak single words (24 months). Developmental retardation became more pronounced in later periods. The patient became bowel and bladder trained at 5 years old. He can not construct complete sentences and can not read at the time of this manuscript preparation (9 years old). Using the New Edition of the Kyoto Scale of Psychological Development, a standardized test widely used in Japan, ${ }^{11}$ we assessed his neurodevelomental status at 36 months and 93 months of age, indicating severe delay in motor (developmental quotient, DQ; 49 and 26, respectively), cognitive (DQ; 62 and 21, respectively), and verbal and social development (DQ; 61 and 23, respectively). Plasma amino acids distributions and plasma concentrations of lactic and pyruvic acids were normal. Computed tomography (2 months and 2 years of age) and magnetic resonance images ( 6 and 8 years of age) of the brain did not demonstrate any abnormalities. At 4 years old, the patient demonstrated a brief, repetitive loss of consciousness. Electroencephalographic findings led to the diagnosis of symptomatic generalized epilepsy presenting with atypical absence. Epileptic symptoms were partially controlled with combined administration of valproric acid, clobazam, and ethosuximide, and remained under observation. Physical growth has failed to thrive. At 9 years old, body height and weight was $121.7 \mathrm{~cm}$ 
(-1.8 SD) and $21.0 \mathrm{~kg}(-1.5 \mathrm{SD})$, respectively. Dental development has also been delayed - first mandibular permanent molars erupted at 8 years of age, and of 8 permanent incisors, only 3 have erupted at 9 years of age.

\section{Mutational analysis and immunoblot analysis of the genes responsible for SCN}

We obtained written informed consent for analysis from both parents. All study centers received approval from the institutional review boards to perform genetic analysis. Mutations in CSF3R, ELA2, and WAS were analyzed by previously described procedures. ${ }^{12-15}$ For sequencing analysis of $H A X 1$, genomic DNA was extracted from the peripheral blood leukocytes. Polymerase chain reaction (PCR) was performed with primers that span all exons of HAX1 (5'-CGTTTACGACAGTGTCAGGATCG-3' and 5'-TGACAAACTGACATGGCCCCAG-3') using the Expand High Fidelity PCR System (Roche Diagnostics GmbH, Mannheim, Germany). The PCR products were sequenced using 9 primers (primer design will be provided upon request). These were then analyzed by cycle sequencing using a BigDye Terminator v3.1 cycle sequencing kit (Applied Biosystems, Foster City, CA) and an ABI PRISM 310 genetic analyzer (Applied Biosystems).

To perform immunoblot analysis of HAX1, cell extracts of peripheral blood leukocytes were subjected into SDS-PAGE. The proteins were transferred to polyvinyldifluoride membranes (Immobilon-P, Millipore, Bradford, MA) using a semi-dry electroblotter (ATTO Corp., Tokyo, Japan). Following the transfer, immunoblots were incubated with a monoclonal antibody against HAX1 (BD Biosciences Pharmingen, Franklin Lakes, NJ). After washing the membranes, the bound secondary anti-mouse antibody was labeled for visualization using SuperSignal (Pierce Biotechnology Inc., Rockford, IL) by enhanced chemiluminescence (FUJIFILM Corp., Tokyo, Japan).

\section{Results and Discussion}

Sequence analysis of the patient demonstrated wild type for CSF3R, ELA2, and WAS. Next, we analyzed HAX1 mutations of the patient, and identified a homozygous single-base pair substitution (256C-to-T) leading to a stop codon at codon 86 (R86X). This deficiency was confirmed by the absence of HAX1 protein in immunoblot analysis. The site of this mutation was consistent with that reported in one of 23 SCN patients in the previous study. ${ }^{7}$ Although neither parent showed a detectable phenotype, both parents had a heterozygous R86X mutation with the expression of HAX1 proteins.

HAX1 has recently been identified as a cause of SCN by Klein et al., who analyzed 23 ELA2 mutation-negative SCN patients. ${ }^{7}$ According to the mutation sites, the 23 patients were classified into 3 groups; W44X in 19 patients mainly in Kurdish and Turkish populations, R86X in 1 Iranian patient, and Q190X in 3 Swedish patients from an original Kostmann family. ${ }^{7}$ One implication of this finding might be that mutation sites are linked to ethnicity. Our patient is the second patient identified as harboring R86X mutation, and the first patient with HAX1-deficient SCN in the Japanese population.

Although not fully understood, HAX1 seems to play a critical role in apoptosis and the organization of cytoskeleton..$^{816-18} H A X 1$ is a ubiquitously expressed human gene, 8 and therefore symptoms other than myelopoietic disorder could be present in patients with this deficiency. Indeed, some of the 23 Klein's patients were associated with conditions other than low ANC.' These included splenomegaly $(n=8)$, growth failure $(n=2)$, muscular hypotonia $(n=1)$, and tricuspid insufficiency $(n=1) .^{7}$ Of these, splenomegaly is a common feature with SCN irrespective of the underlying genetic defects. ${ }^{1}$ Our patient has failed to thrive, a condition concordant with the finding in the first patient showing the same R86X mutation. ${ }^{7}$ Together with dental development delay, this may be a reflection of a malfunction in cytoskeleton organization due to HAX1 deficiency. ${ }^{8}$

Of special note is the finding that our patient showed severe delay in motor and intellectual milestones. Several explanations of such developmental delay exist. One possibility is derived from hearing impairment due to sequelae at the first presentation of otomastoiditis. However, since hearing impairment was moderate and unilateral, it is unlikely that this was the cause of severe developmental delay, and is, at least, unrelated to the delay of motor function. The second possibility is that infection due to initial otomastoiditis might have extended to the central nervous system. However, this possibility is unlikely because the patient's general condition had remained well and brain CT scans at that time did not show any abnormalities. Thus, the most plausible explanation is attributable to $H A X 1$ mutation. This explanation is supported by the report ${ }^{19}$ by Carlsson and Fasth, who described neurological impairments in 3 of $6 \mathrm{SCN}$ patients of the original Kostmann pedigree; some of these patients were later demonstrated to be HAX1-deficient. ${ }^{7}$ Of the three, one had mild mental retardation, the second showed speech and gross motor retardation, and the third demonstrated attention deficit hyperactivity syndrome plus epilepsy. ${ }^{19}$ Furthermore, an experimental study showed that HAX1 is maximally expressed in brain tissues on Western blot analysis. ${ }^{9} \mathrm{~N} 2 \mathrm{a}$ cells, a mouse neural cell line, also contained significant amount of HAX1 mRNA with subcellular localization in the cytoplasm and mitochondria. ${ }^{9}$ Collectively, HAX1 deficiency may potentially affect neural development, reflecting severe developmental retardation in our patient. However, whether neurological complication in our patient is linked to mutation sites in HAX1 or to HAX1 deficiency itself is not clear. 
In addition, since diverse proteins have recently been identified to interact with HAX1, ${ }^{8,16-18}$ some of these or other yet to be determined genetic factor(s) may be involved in this condition. Further accumulation of similar patients is needed to clarify this question.

In conclusion, we presented a sporadic SCN patient with a nonsense mutation in HAX1. Importantly, this is the first case in the Japanese population and the second patient with an R86X mutation. Furthermore, this patient demonstrated severe developmental delay and epilepsy.

K. Matsubara, K. Imai, S. Okada, M. Miki, N. Ishikawa, M. Tsumura, T. Kato, O. Ohara, S. Nonoyama, M. Kobayashi

Department of Pediatrics, Nishi-Kobe Medical Center, Kobe (KM), Department of Pediatrics, National Defense Medical College, Tokorozawa, Saitama (KI, SN), Department of Pediatrics, Hiroshima University Graduate School of Biomedical Sciences, Hiroshima (SO, MM, NI, MT, MK), and Department of Human Genome Technology, Kazusa DNA Research Institute, Kisarazu, Chiba (TK, OO), Japan

Correspondence: Kousaku Matsubara, MD, PhD, Department of Pediatrics, Nishi-Kobe Medical Center, 5-7-1 Kojidai, Nishi-ku, Kobe 651-2273, Japan.

Tel: +81-78-997-2200, Fax: +81-78-993-3728

E-mail:kskmatsu@s4.dion.ne.jp

Key words: developmental delay, epilepsy, HAX1 deficiency, severe congenital neutropenia

Acknowledgments: We thank Dr. S. Okamura, First Department of Internal Medicine, Faculty of Medicine, Kyushu University, Fukuoka, Japan, for analyzing mutations in CSF3R gene. This study was supported in part by Grants from Japan Intractable Diseases Research Foundation, Kawano Masanori Foundation for Promotion of Pediatrics.

Authors' Contributions: KM was responsible for clinical management and wrote the manuscript. KI contributed to the design of the study, genetic analysis, and drafting and editing of the manuscript. SO, $M M, N I$, and MT carried out genetic analysis and immunoblotting. TK and OO carried out genetic analysis. SN and MK designed the study and critically reviewed the manuscript. All authors approved the version submitted for publication.

\section{References}

1. Welte K, Zeidler C, Dale DC. Severe congenital neutropenia. Semin Hematol 2006; 43:189-95.

2. Kostmann R. Infantile genetic agranulocytosis. A new recessive lethal disease in man. Acta Paediatr Scand 1956; 45:1-78.

3. Dong F, Brynes RK, Tidow N, Welte K, Löwenberg B, Touw IP.
Mutations in the gene for the granulocyte colony-stimulatingfactor receptor in patients with acute myeloid leukemia preceded by severe congenital neutropenia. N Engl J Med 1995; 333:487-93.

4. Ancliff PI, Gale RE, Liesner R, Hann IM, Linch DC. Mutations in the ELA2 gene encoding neutrophil elastase are present in most patients with sporadic severe congenital neutropenia but only in some patients with the familial form of the disease. Blood 2001; 98:2645-50.

5. Devriendt K, Kim AS, Mathijs G, Frints SG, Schwartz M, Van Den Oord JJ, et al. Constitutively activating mutation in WASP causes X-linked severe congenital neutropenia. Nat Genet 2001; 27:313-7.

6. Person RE, Li FQ, Duan Z, Benson KF, Wechsler J, Papadaki $\mathrm{HA}$, et al. Mutations in proto-oncogene GFI1 cause human neutropenia and target ELA2. Nat Genet 2003; 34:308-12.

7. Klein C, Grudzien M, Appaswamy G, Germeshausen M, Sandrock I, Schäffer AA, et al. HAX1 deficiency causes autosomal recessive severe congenital neutropenia (Kostmann disease). Nat Genet 2007; 39:86-92.

8. Suzuki Y, Demoliere C, Kitamura D, Takeshita D, Deuschle U, Watanabe T. HAX-1, a novel intracellular protein, localized on mitochondria, directly associates with HS1, a substrate of Src family tyrosine kinases. J Immunol 1997; 158:2736-44.

9. Hippe A, Bylaite M, Chen M, von Mikecz A, Wolf R, Ruzicka $\mathrm{T}$, et al. Expression and tissue distribution of mouse Hax1. Gene 2006; 379:116-26.

10. Matsubara K, Omori K, Baba K. Acute coalescent mastoiditis and acoustic sequelae in an infant with severe congenital neutropenia. Int J Pediatr Otorhinolaryngol 2002; 62:63-7.

11. Maehara T, Shimizu H, Kawai K, Shigemoto R, Tamagawa K, Yamada T, et al. Postoperative development of children after hemispherotomy. Brain Dev 2002; 24:155-60.

12. Yokoyama T, Okamura S, Asano Y, Kamezaki K, Numata A, Kakumitsu $\mathrm{H}$, et al. A novel mutation in the juxtamembrane intracellular sequence of the granulocyte colony-stimulating factor (G-CSF) receptor gene in a patient with severe congenital neutropenia augments GCSF proliferation activity but not through the MAP kinase cascade. Int J Hematol 2005; 82:28-34.

13. Imai $K$, Morio $T$, Zhu $Y$, Jin $Y$, Itoh $S$, Kajiwara $M$, et al. Clinical course of patients with WASP gene mutations. Blood 2004; 103:456-64

14. Kawaguchi H, Kobayashi M, Nakamura K, Konishi N, Miyagawa S, Sato T, et al. Dysregulation of transcriptions in primary granule constituents during myeloid proliferation and differentiation in patients with severe congenital neutropenia. J Leukoc Biol 2003; 73:225-34.

15. Sera Y, Kawaguchi H, Nakamura K, Sato T, Habara M, Okada $\mathrm{S}$, et al. A comparison of the defective granulopoiesis in childhood cyclic neutropenia and in severe congenital neutropenia. Haematologica 2005; 90:1032-41.

16. Radhika V, Onesime D, Ha JH, Dhanasekaran N. Galpha13 stimulates cell migration through cortactin-interacting protein Hax-1. J Biol Chem 2004; 279:49406-13.

17. Cilenti L, Soundarapandian MM, Kyriazis GA, Stratico V, Singh S, Gupta S, et al. Regulation of HAX-1 anti-apoptotic protein by Omi/HtrA2 protease during cell death. J Biol Chem 2004; 279:50295-301.

18. Han Y, Chen YS, Liu Z, Bodyak N, Rigor D, Bisping E, et al. Overexpression of HAX-1 protects cardiac myocytes from apoptosis through caspase-9 inhibition. Circ Res 2006; 99:415-23.

19. Carlsson G, Fasth A. Infantile genetic agranulocytosis, morbus Kostmann: presentation of six cases from the original "Kostmann family" and a review. Acta Paediatr 2001; 90:757-64. 